\title{
Interactive comment on "The impact of shoreline change on the salinity distribution in the wetlands of Liao River Estuary, China" by Mingliang Zhang et al.
}

\section{Anonymous Referee \#3}

Received and published: 24 September 2020

\section{General comments}

This manuscript describes a series of FVCOM experiments that aim to investigate the sensitivity of salinity distribution in Liao River Estuary to the construction of the Panjin Port. Motivation is driven in part by considering that the survivability of two species of salt-tolerant plants in the LRE may be negatively affected.

The model setup section is in need of an overhaul to better describe what has been done here (details below), and more broadly the manuscript should receive some reorganization to get all of the right content into the right sections. For example the start of section 4.1 has introduction material (pg 12, lines 1-5), and section 4.2 also has in- 
troduction material (pg 14, lines 8-18), while at pg 14, lines 18-21 look like they belong in a methods section.

OSD

Despite the organizational issues, the manuscript starts off with a focused goal of investigating salinity distribution sensitivity and demonstrates/quantifies the effects of adjusting the domain geometry. In that regard it is successful. Surface forcing is absent so there is a lack of completeless for the model, but this is noted for future work, and presumably explains why specific forecasts about the fate of the salt-tolerant plants is not made.

\section{Specific comments}

pg 7, lines 17-18: What is the "validated Bohai Sea Parent Model"? Definitely we need some sort of citation here, and some rationale for using it's water level as OBC in the present study. Does this model provide tides only or does it also include non-tidal sea-level variability?

pg 7, lines 18-19: "The open boundary for salinity was set to 34 PSU at the sea surface and interpolated along the sigma layers." I don't follow; more than one number is needed to conduct an interpolation. Does this mean the salinity OBC was set to 34 PSU at all depths along the entire open boundary? Is the 34 PSU isohaline known to coincide with the model open boundary? How does that reconcile with estuarine flow characterised by salty inflow at depth and fresher outflow in upper layers?

pg 7, lines 19-20: "Temperature was set to $15^{\circ} \mathrm{C}$ across the whole domain." This sounds like the temperature initial condition. What was done for the temperature OBC? Was temperature an active or inactive tracer in the model? Is water temp mostly uniform in the area justifying setting it to inactive in the model?

pg 7, lines 20-21: "The initial condition for salinity was based on the steady results derived by running the model for approximately four months." This reads as if the final conditions were used to initialize the model! Presumably the model was started with a
Interactive comment
Printer-friendly version

Discussion paper 
different set of conditions (perhaps uniform salinity?) and then the four month run used (perhaps with average runoff?) to produce quasi steady salinity field for initializing the OSD four cases. Please clarify this.

pg 7: No information provided about velocity boundary conditions.

pg 7: No justification for not including surface forcing.

Interactive

comment

pg 7, lines 21-23: How were these numbers selected? Are there discharge records that were used? pg6 line 3 mentions low average and high averages of $101 \& 285$ $\mathrm{m} 3 / \mathrm{s}$ for LR but a value of 25 is used for cases $1 \& 2$. Some rationale for the choices would help here.

pg 8, lines 12-15: It is typical to evaluate water level by splitting into tidal and residual/sub-tidal components, this would help in understanding if mismatches are due to to poorly tuned tides or due to poor non-tidal ssh from the forcing model.

pg 9, lines 10-11: How was 30 minutes of phase lag measured? Is this associated with a particular tidal constituent?

pg 9, lines 13-23 and Figures 5,6: Terminology switches between flow speeds and tidal flow. Suggest to be precise here, tidal currents are typically extracted via tidal analysis and there is no mention of such analysis.

pg 17: How was the residual circulation calculated? Through an average or through a detiding procedure?

Figure 15: vectors are very dense, consider replotting with fewer vectors so the current field is more visible

Figure 15: there is a gap in vectors along the Daliao River; is that a plotting artifact?

Printer-friendly version

pg 19, line 14: "The ecological degradation of wetlands in the LRE has become more and more severe in the past decade." This line difficult to reconcile with the first line of the abstract ("The wetland of Liao River Estuary in northeast China is one of the 
best-preserved wetlands across the globe")

pg 19, line 6,7: The salinity appears to vary considerably between dry and wet season

OSD at PBW, is a 4 PSU increase in the wet season enough to affect the species here?

Technical corrections

pg 7, line 11: which DEMs were used?

Interactive

comment

pg 7, line 16-17: are internal and external switched here?

pg 8: Which simulation was validated? Presumably it was case 1 or 3 ?

Interactive comment on Ocean Sci. Discuss., https://doi.org/10.5194/os-2020-36, 2020. 